\title{
Comparison of LncRNA Expression Profiles during Myogenic Differentiation and Adipogenic Transdifferentiation of Myoblasts
}

\author{
Renli Qi ${ }^{1, *,+}$, Xiaoyu Qiu ${ }^{2,+}$, Yong Zhang ${ }^{3}$, Jing Wang ${ }^{2}$, Qi Wang ${ }^{1}$, Min Wu ${ }^{1}$, Jinxiu Huang ${ }^{1}$ \\ and Feiyun Yang ${ }^{2, *}$ \\ 1 Animal Nutrition Institute, Chongqing Academy of Animal Science, Rongchang, Chongqing 40240, China \\ 2 Key Laboratory of Pig Industry Sciences, Ministry of Agriculture, Rongchang, Chongqing 402460, China \\ 3 School of Life Science and Engineering, Southwest University of Science and Technology, \\ Mianyang 621010, China \\ * Correspondence: qirenli999@sina.com (R.Q.); yfeiyun@yeah.net (F.Y.); Tel.: +86-023-46792097 (R.Q.); \\ +86-023-46792056 (F.Y.) \\ + These authors contribute equally to this work.
}

Received: 27 June 2019; Accepted: 28 July 2019; Published: 30 July 2019

\begin{abstract}
Myoblasts could transdifferentiate into adipocytes or adipocyte-like cells, which have the capability of producing and storing intracellular lipids. Long-chain non-coding RNAs (lncRNAs) have many important physiological functions in eukaryotes, which include regulating gene expression, chromosome silencing, and nuclear transport. However, changes in the expression of lncRNAs in muscle cells during adipogenic transdifferentiation have not been investigated to date. Here, C2C12 myoblasts were seeded and then induced to undergo myogenic and adipogenic transdifferentiation. The expression profiles of IncRNAs in various differentiated cells were analyzed and then compared by digital gene expression (DGE) RNA sequencing. A total of 114 core lncRNAs from 836 differentially expressed lncRNAs in adipogenic cells were identified. Further investigation by in silico analysis revealed that the target genes of core lncRNAs significantly enriched various signaling pathways that

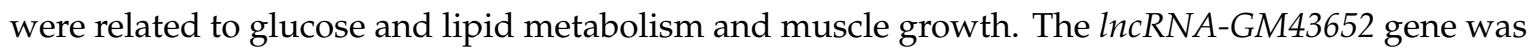
a potential regulator of adipogenesis in muscle cells. It showed the highest levels of expression in adipogenic cells, and the knocking down $\operatorname{lnc} R N A-G M 43652$ negatively influenced lipid deposition in transdifferentiated myoblasts. This study has identified the novel candidate regulators that may be assessed in future molecular studies on adipogenic conversion of muscle cells.
\end{abstract}

Keywords: transdifferentiation; adipogenesis; lncRNA; myoblasts; RNA sequencing; C2C12 cells

\section{Introduction}

Transdifferentiation is the transformation of one cell type to another. Myoblasts have the potential to transdifferentiate into adipocytes or adipocyte-like cells under specific induction conditions (i.e., drug stimulation, cytokine treatment) [1-6]. Skeletal muscle satellite cells in humans and animals retain broad differentiation capacity, including the generation of adipocytes in an adipogenic medium in a process that the insulin sensitizer-rosiglitazone-enhances [7]. Myogenic C2C12 cells also can be converted to adipocytes by overexpression of $\operatorname{PPAR} \gamma$ and $\mathrm{C} / \mathrm{EBP} \alpha$, which are two critical mediators for cellular adipogenesis [1,8]. A few skeletal muscle cells could be replaced by fat cells during regeneration in humans [9]. During skin wound healing in mice, the regeneration of hair follicles is accompanied by the transformation of numerous myofibers (myofibroblasts) into white adipocytes [10]. The BMP signaling pathway is activated, which in turn drives the transformation of "myofibroblast-adipocytes" [10]. 
Adipogenic transdifferentiated muscle cells lose their myogenic ability, but they gain the adipogenic ability to produce and store lipids within cells. The conversion usually occurs in muscle progenitor or satellite cells [3]. The multinucleated myocytes (myotubes) at terminal differentiation do not have the ability to transdifferentiate. The shift between muscle and adipose tissues results in changes in organ function and metabolism. However, to date, the molecular mechanism underlying the conversion of muscle-adipose is not fully understood.

In recent years, non-coding RNAs (ncRNAs) have become a research topic of interest in the life sciences. Various ncRNAs (e.g., miRNAs, ceRNAs, and lncRNAs) are essential for gene transcription, cleavage, translation, modification, and expression, and therefore exhibit a wide range of regulatory roles in different life events [11-13]. Some coding genes and non-coding genes (particularly miRNAs) are involved in the control of adipogenic transdifferentiation of myocytes [14-16]. However, most of the ncRNAs in humans and animals are long-chain ncRNAs (lncRNAs), with lengths of more than $200 \mathrm{nt}$. LncRNAs commonly have complex molecular structures and diverse functions, such as the control of cell fate decision, cycle, proliferation, differentiation, and apoptosis [17-19]. Several IncRNAs have been known for their important regulatory roles in the formation and physiological function of muscle and fat tissues, such as LncMyoD, Linc-YY1, Blnc1, and Lnc-ORA [20-24]. For instance, the expression of the lncRNA slincRAD is unregulated during the early differentiation stages of 3T3-L1 adipocytes. The abolishment of the interaction between slincRAD and the DNMT1 gene will result in defective epigenetic regulation, which in turn compromises adipogenesis [25]. However, the expression and function of lncRNAs in the adipogenic transdifferentiation of muscle cells have not been investigated to date.

In this study, we aimed to identify the lncRNAs that are involved in the adipogenic transformation of muscle cells to reveal the molecular mechanism of transdifferentiation. $\mathrm{C} 2 \mathrm{C} 12$ is a murine myoblasts cell line that has been used as a classic cell model for the study of muscle growth, development, and function [26]. Here, C2C12 cells were induced to undergo myogenic differentiation and adipogenic transdifferentiation. Subsequently, the expression profiles of lncRNAs in the various differentiated cells were assessed by RNA-seq-based digital gene expression (DGE-Seq) analysis [27,28]. A total of 114 significant differentially expressed (DE) lncRNAs (RPKM $>100$, fold-change $>2$, and $P$-value $<0.001$ ) between the myogenic cells and the adipogenic cells were obtained, and their target mRNAs were

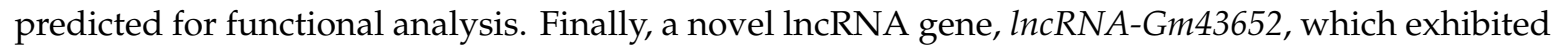
the highest expression in adipogenic differentiated cells, was determined to promote adipogenesis in muscle cells. These results provide useful information that may be utilized in the elucidation of the molecular mechanism of adipogenic transdifferentiation of muscle cells.

\section{Results}

\subsection{The Adipogenic Transdifferentiation of $\mathrm{C} 2 \mathrm{C} 12$ Myoblasts}

Figure 1A shows that the cells are spirally arranged and they gradually fuse into multinuclear cells with the normal myogenic induction, and then into formatted myotubes after six days of induction. The adipogenic transdifferentiated cells gradually became round via adipogenic induction, with numerous small lipid droplets inside the cells. qRT-PCR detected the key regulatory factors that are related to myogenesis or adipogenesis in different cells (Figure 1B,C). This observation is in line with the fact that $F A S$ and PPAR $\gamma$, which are two key adipogenesis markers, exhibit upregulated mRNA expression levels in adipogenic differentiated cells (ADCs) as compared to undifferentiated cells (UDCs) during cellular adipogenesis. The myogenesis regulators, Myf5 and myogenin, showed stable high expression in the myogenic differentiated cells (MDCs). However, the number and size of lipid droplets in the adipogenic myoblasts were relatively fewer and smaller than those of mature adipocytes at the same differentiation induction time (Supplementary Figure S1A). The brown adipocyte-specific genes, UCP1 and PRDM16, both showed higher expression levels in the ADCs when compared to the 3t3-L1 
adipocytes (Supplementary Figure S1B). This suggests that the adipogenic cells that had transformed from myoblasts possess the brown adipocyte signatures.

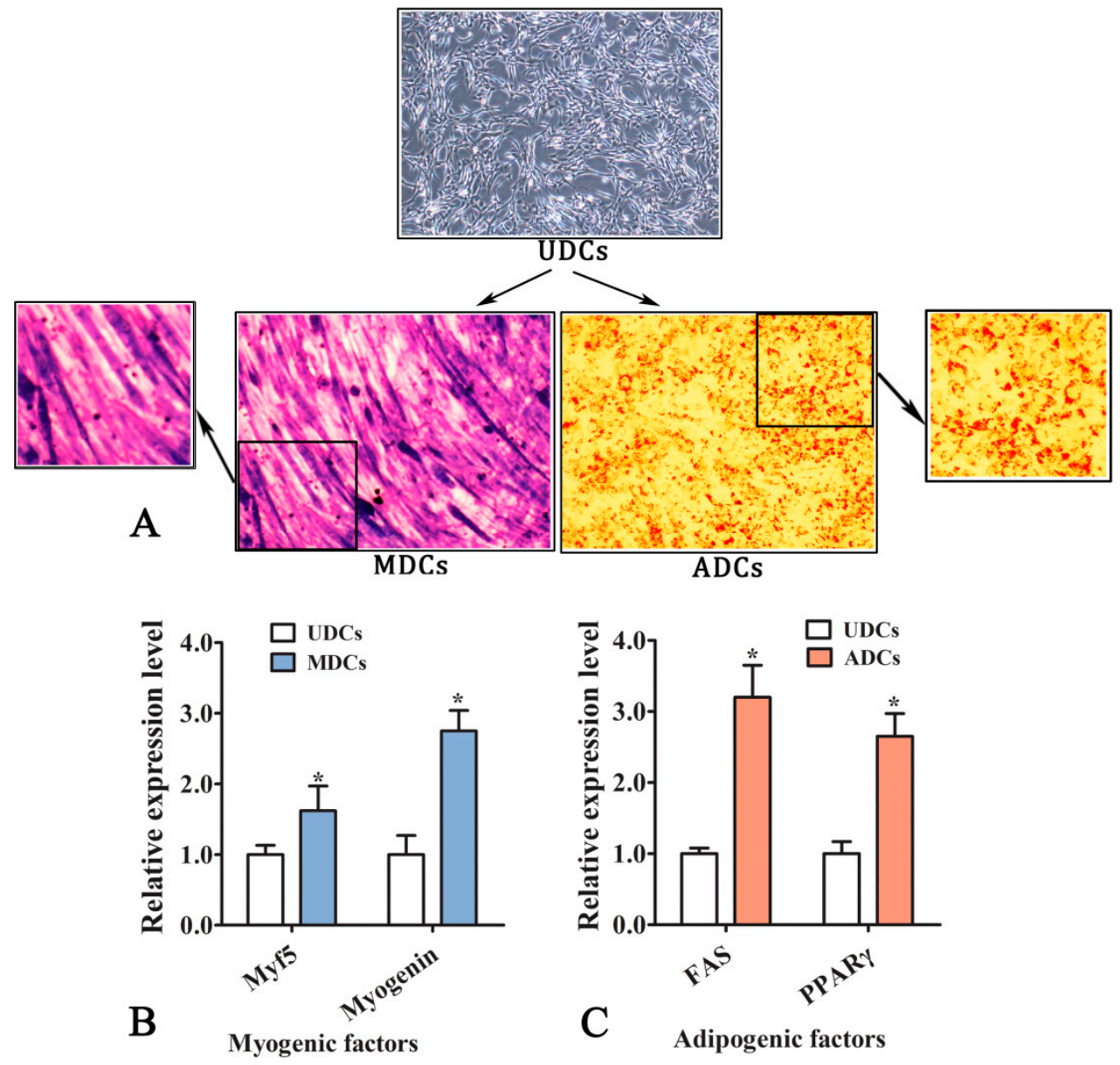

Figure 1. The myogenic differentiation and the adipogenic transdifferentiation of $\mathrm{C} 2 \mathrm{C} 12$ myoblasts. (A) Morphological difference between adipogenic differentiated cells (ADCs) and myogenic differentiated cells (MDCs). MDCs are stained with Giemsa after six days of the myogenesis differentiation induction, and ADCs are stained with Oil red $\mathrm{O}$ after eight days of the adipogenesis differentiation induction. UDCs, undifferentiated cells. (B) Relative mRNA levels of the myogenic genes in MDCs and UDCs. (C) Relative mRNA levels of the adipogenic genes in ADCs and UDCs. The expression levels were detected by qRT-PCR, $n=5$. The data are presented as the means \pm SEM. * $P \leq 0.05$, student's $t$-test.

\subsection{DGE-RNA Sequencing of $\operatorname{lncRNAs}$}

High-throughput DGE-RNA sequencing was used to assess the expression of lncRNAs and protein-coding RNAs in UDCs, ADCs, and MDCs to reveal the differential expression of lncRNAs among various cells. Total RNA was extracted from the cells for preparation of RNA libraries. Three RNA sequencing libraries were sequenced on the Illumina ${ }^{\circledR}$ HiSeq 2500 platform. Cufflinks and Scripture assembled a total of 48,615 known protein-coding gene transcripts (for 17,568 mRNAs) and 8594 known lncRNA transcripts (for 6363 lncRNAs) by comparing the clean sequencing data with the current mouse genome.

Figure 2A shows the holistic expression levels of mRNAs and lncRNAs in different cells. The average expression level of the lncRNAs was slightly higher in the differentiated cells relative to the undifferentiated cells. Additionally, no clear difference in the average expression level between lncRNAs and mRNAs was observed. Transcript types and the chromosomal distribution of lncRNAs 
were determined (Figure 2B,C). The comparison of transcript length and exon number of the two transcripts are shown in Figure 2D,E. Figure 2F shows that most of the lncRNAs contained a relatively shorter ORF when compared with that of the mRNAs.

Low-expression, single-exon, employing a read coverage threshold, unreliable fragments, and annotated non-lncRNA transcripts (e.g., annotated protein-coding genes, pre-microRNAs, tRNAs, rRNAs, and pseudogenes) and transcripts with coding potential were eliminated. Finally, 4685, 3578, and 3487 rigorous IncRNA transcripts were, respectively, obtained from the UDCs, ADCs, and MDCs.

Table 1 shows the top 20 lncRNAs that are expressed in the three different cells based on RPKM. Similar to housekeeping genes, some lncRNAs are highly expressed in all the three different cells, which include ENSMUST00000192833, ENSMUST00000182520, and ENSMUST00000174808. However, several lncRNAs exhibited cell type-specific expression. For example, ENSMUST00000196219 and ENSMUST00000180396 were only highly expressed in the ADCs.
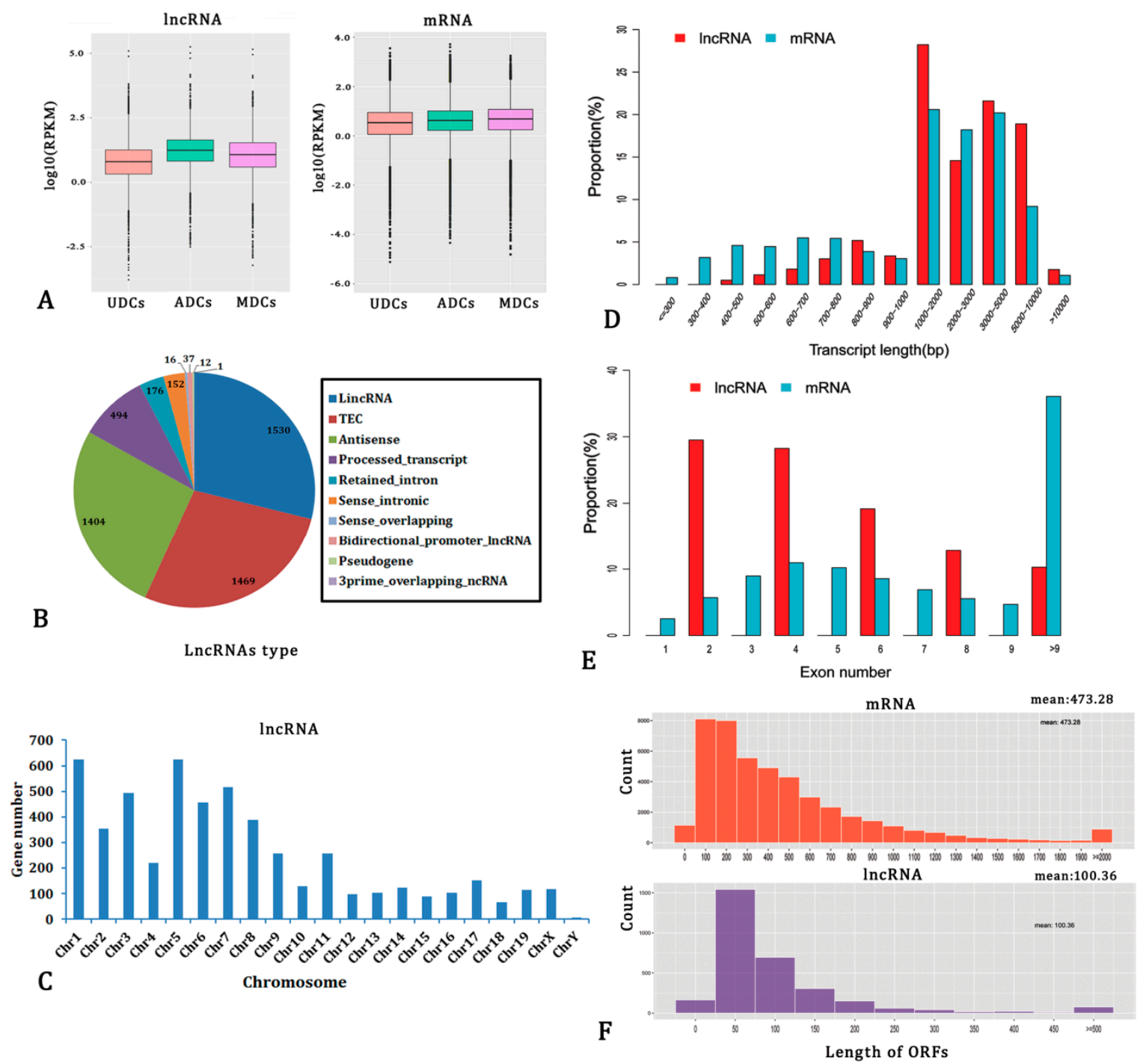

Figure 2. The features of long non-coding RNA (lncRNAs) and mRNAs. (A) The FPKM distribution of all identified lncRNAs and mRNAs at three different cells. (B) Percentage of different types of lncRNAs. (C) Distribution of lncRNA transcripts along each chromosome. (D) Transcript size distribution of lncRNAs and mRNAs. (E) Exon number per transcript of lncRNAs and mRNAs. (F) Distribution of the open reading frame (ORF) length of lncRNAs and mRNAs. 
Table 1. The top expressed 20 lncRNA transcripts in the UDCs, MDCs, and ADCs.

\begin{tabular}{cccccc}
\hline UDCs & & ADCs & & MDCs \\
\hline lncRNAs & RPKM & lncRNAs & RPKM & lncRNAs & RPKM \\
\hline ENSMUST00000192833 & $124,640.19$ & ENSMUST00000196219 & $180,327.40$ & ENSMUST00000192833 & $145,216.23$ \\
ENSMUST00000182520 & $77,450.29$ & ENSMUST00000192833 & $104,112.48$ & ENSMUST00000182520 & $90,764.06$ \\
ENSMUST00000174808 & 6608.49 & ENSMUST00000182520 & $65,232.66$ & ENSMUST00000174808 & $14,136.14$ \\
ENSMUST00000139288 & 5952.70 & ENSMUST00000172812 & $15,463.37$ & ENSMUST00000173314 & $12,237.22$ \\
ENSMUST00000172812 & 5176.44 & ENSMUST00000174808 & $13,599.96$ & ENSMUST00000172812 & $11,376.69$ \\
ENSMUST00000173314 & 5173.88 & ENSMUST00000173314 & $12,106.39$ & ENSMUST00000181751 & 3401.46 \\
ENSMUST00000209681 & 4609.90 & ENSMUST00000173499 & 6486.05 & ENSMUST00000174784 & 3368.69 \\
ENSMUST00000126640 & 4586.59 & ENSMUST00000148202 & 6416.90 & ENSMUST00000173523 & 3034.68 \\
ENSMUST00000123278 & 4550.22 & ENSMUST00000180396 & 6392.42 & ENSMUST00000173499 & 2907.27 \\
ENSMUST00000181751 & 4292.44 & ENSMUST00000173523 & 5893.02 & ENSMUST00000139288 & 2581.09 \\
ENSMUST00000187415 & 4249.52 & ENSMUST00000198476 & 5307.10 & ENSMUST00000192176 & 2570.33 \\
ENSMUST00000118575 & 3575.11 & ENSMUST00000174784 & 5116.32 & ENSMUST00000140716 & 2219.49 \\
ENSMUST00000148202 & 3258.77 & ENSMUST00000209541 & 4304.59 & ENSMUST00000148202 & 2035.23 \\
ENSMUST00000193796 & 3055.50 & ENSMUST00000122365 & 4108.33 & ENSMUST00000123278 & 2018.85 \\
ENSMUST00000192629 & 2613.42 & ENSMUST00000192176 & 3958.38 & ENSMUST00000209681 & 2012.44 \\
ENSMUST00000211359 & 2580.81 & ENSMUST00000181751 & 3701.19 & ENSMUST00000126640 & 2000.11 \\
ENSMUST00000146654 & 2203.23 & ENSMUST00000182010 & 2920.89 & ENSMUST00000187415 & 1903.12 \\
ENSMUST00000134427 & 2191.40 & ENSMUST00000187351 & 2389.74 & ENSMUST00000182010 & 1777.71 \\
ENSMUST00000181631 & 2063.67 & ENSMUST00000072769 & 2353.86 & ENSMUST00000152754 & 1735.28 \\
ENSMUST00000192994 & 2042.13 & ENSMUST00000176549 & 2237.67 & ENSMUST00000136359 & 1727.49 \\
\hline
\end{tabular}

\subsection{DE $\operatorname{lnc} R N A s$}

The heatmap of DE lncRNAs showed that the expression profiles of lncRNAs in the three cell types exhibited extreme significant differences (Figure 3A). KCL cluster analysis indicated that the expression profiles of lncRNAs were more similar in the UDCs and MDCs, whereas the ADCs showed a distinct gene expression pattern.

Huge differences in lncRNA expressions were observed between ADCs and MDCs (Figure 3B-D). Approximately 711 lncRNAs were specifically expressed in MDCs and 803 lncRNAs were only expressed in ADCs. However, most of the cell-specific lncRNAs exhibited very low expression $(\mathrm{RPKM}<1)$. We further focused on the DE lncRNAs between the MDCs and ADCs. A total of 836 lncRNA showed significant differences in expression levels between ADCs and MDCs $(t$-test, FDR $<0.05)$. In Figure 3E, except for the low-expression genes (RPKM $<100$ ), there were still 43 lncRNAs that were significantly upregulated and 71 lncRNA transcripts were significantly downregulated in ADCs (FDR $<0.001$ and fold-change $>2$ ). These 114 significant DE lncRNAs were considered to be core lncRNAs, which possibly function as regulators of adipogenesis of myoblasts. Figure $3 \mathrm{~F}$ show the top 10 upregulated and 10 downregulated lncRNA transcripts.

Moreover, a total of 1065 protein-coding genes showed altered expression levels in ADCs relative to MDCs (FDR < 0.01), of which 171 mRNAs were significant differential expression, with FDR $<0.001$ and fold change $>2$ (Supplementary Figure S1A). Nine mRNAs transcripts (ENSMUST00000023934, ENSMUST00000098192, ENSMUST00000153218, ENSMUST00000026907, ENSMUST00000112832, ENSMUST00000040153, ENSMUST00000165430, ENSMUST00000144897, and ENSMUST00000037023) showed an extreme increase in ADCs $(\log 2 \mathrm{FC}>5)$, which indicated that these mRNAs control or affect lipogenesis in muscle cells. Table 2 shows the top 30 mRNA transcripts exhibiting significant changes in expression.

KEGG pathway analysis indicated that the 171 altered mRNA transcripts enriched several pathways that were related to nutrient metabolism in cells such as tyrosine $(P=0.035)$, glycolysis $(P=0.049)$, and fructose metabolism $(P=0.098)$ (Supplementary Figure $S 2)$. These findings suggest that the distinct differentiation of cells determine the variations in intracellular nutrient metabolism and utilization. 


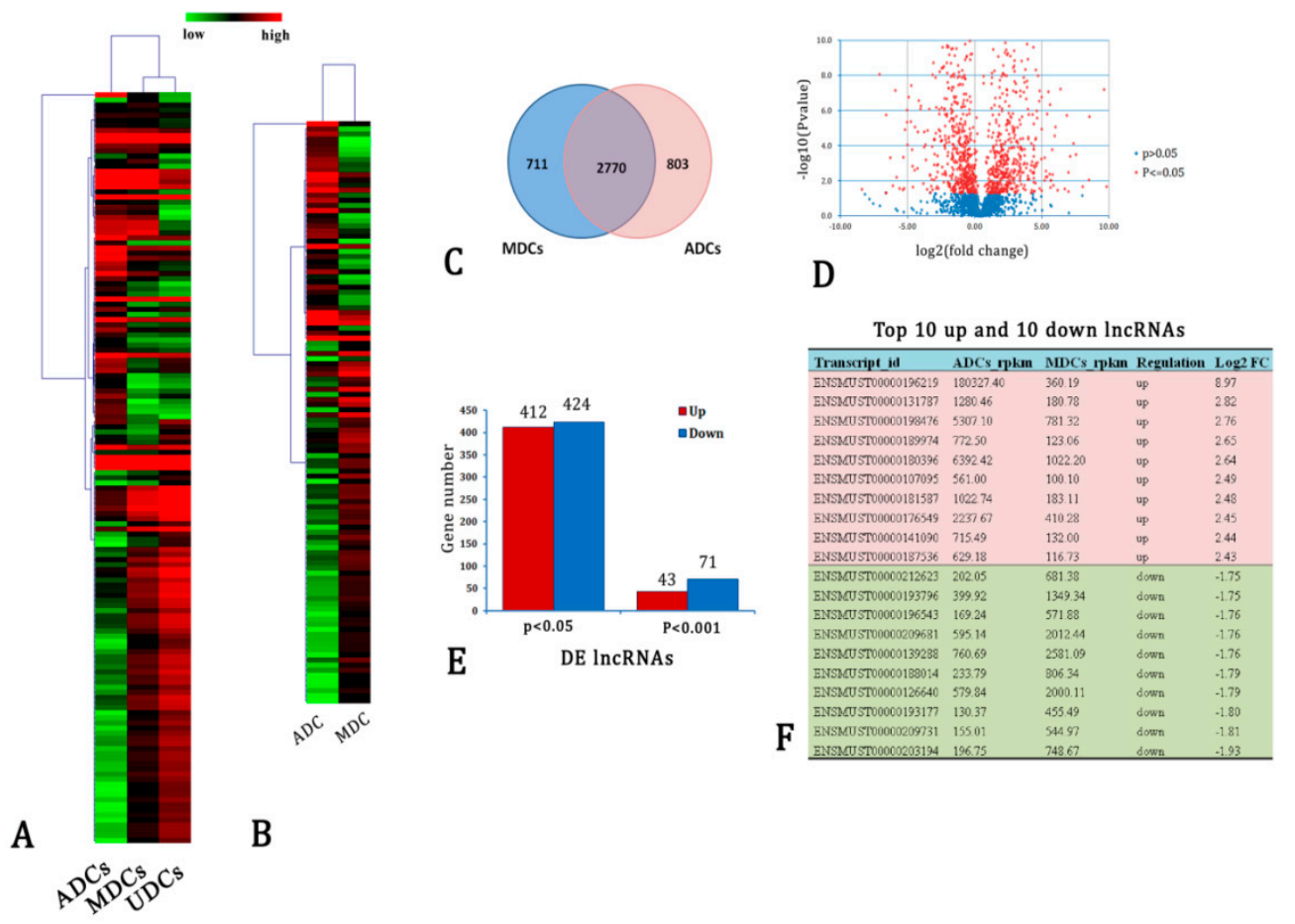

Figure 3. Differentially expressed (DE) lncRNA transcripts in adipogenic differentiated cells (ADCs)/myogenic differentiated cells (MDCs). (A) Heatmap showing that ADCs exhibit a distinct lncRNA expression profile relative to MDCs and UDCs. Red indicates high expression levels, and green represents low expression levels. (B) Heatmap of DE lncRNAs in ADCs/MDCs. (C) Venn diagram of all expressed lncRNAs in ADCs/MDCs. (D) Volcano plot of the DE lncRNAs. Red indicates $P \leq 0.05$. (E) Number of DE lncRNAs with $P \leq 0.05$ and $P \leq 0.001$. (F) Top 10 upregulated and top 10 downregulated lncRNAs in ADCs/MDCs.

Table 2. The top 30 changed mRNA transcripts in ADCs vs. MDCs.

\begin{tabular}{ccccccc}
\hline Accession & ADCs_rpkm & MDCs_rpkm & Regulation & log2 FC & FDR & Gene Symbol \\
\hline ENSMUST00000000466 & 503.48 & 89.23 & up & 2.50 & 0.0000 & Plin2 \\
ENSMUST00000000756 & 384.21 & 96.18 & up & 2.00 & 0.0000 & Rpl13 \\
ENSMUST00000004072 & 1336.05 & 278.51 & up & 2.26 & 0.0000 & Rpl8 \\
ENSMUST00000008036 & 681.50 & 233.72 & up & 1.54 & 0.0000 & Rplp1 \\
ENSMUST00000008812 & 826.36 & 176.26 & up & 2.23 & 0.0000 & Rps18 \\
ENSMUST00000009039 & 512.00 & 198.39 & up & 1.37 & 0.0000 & Rpl30 \\
ENSMUST00000017548 & 856.60 & 192.56 & up & 2.15 & 0.0000 & Rpl19 \\
ENSMUST00000017610 & 168.79 & 463.18 & down & -1.46 & 0.0000 & Timp2 \\
ENSMUST00000018437 & 459.23 & 126.45 & up & 1.86 & 0.0000 & Pfn1 \\
ENSMUST00000020238 & 953.64 & 279.12 & up & 1.77 & 0.0000 & Hsp90b1 \\
ENSMUST00000020640 & 572.53 & 199.28 & up & 1.52 & 0.0000 & Rack1 \\
ENSMUST00000020909 & 155.75 & 471.23 & down & -1.60 & 0.0000 & Laptm4a \\
ENSMUST00000021822 & 27.18 & 341.94 & down & -3.65 & 0.0000 & Ogn \\
ENSMUST00000021933 & 576.35 & 233.46 & up & 1.30 & 0.0000 & Ctsl \\
ENSMUST00000022704 & 251.33 & 644.66 & down & -1.36 & 0.0000 & Itm2b \\
ENSMUST00000023269 & 553.91 & 167.99 & up & 1.72 & 0.0000 & Rpl24 \\
ENSMUST00000023934 & 1074.34 & 0 & up & Inf & 0.0000 & Hbb-bs \\
ENSMUST00000025052 & 350.69 & 68.09 & up & 2.36 & 0.0000 & Rps10 \\
ENSMUST00000025511 & 339.15 & 36.65 & up & 3.21 & 0.0000 & Rps14 \\
ENSMUST00000025563 & 2703.24 & 1266.10 & up & 1.09 & 0.0000 & Fth1 \\
ENSMUST00000026565 & 148.88 & 364.95 & down & -1.29 & 0.0000 & Ifitm3 \\
\hline
\end{tabular}


Table 2. Cont.

\begin{tabular}{ccccccc}
\hline Accession & ADCs_rpkm & MDCs_rpkm & Regulation & log2 FC & FDR & Gene Symbol \\
\hline ENSMUST00000026907 & 1200.90 & 2.96 & up & 8.66 & 0.0000 & Klkb1 \\
ENSMUST00000027409 & 521.98 & 232.54 & up & 1.17 & 0.0000 & Des \\
ENSMUST00000028222 & 364.77 & 99.43 & up & 1.88 & 0.0000 & Hspa5 \\
ENSMUST00000029722 & 1196.45 & 427.35 & up & 1.49 & 0.0000 & Rps3a1 \\
ENSMUST00000031318 & 17.93 & 417.80 & down & -4.54 & 0.0000 & Cxcl5 \\
ENSMUST00000031617 & 350.05 & 135.61 & up & 1.37 & 0.0000 & Rp16 \\
ENSMUST00000032934 & 356.74 & 154.31 & up & 1.21 & 0.0000 & Aldoa \\
ENSMUST00000033683 & 577.73 & 200.73 & up & 1.53 & 0.0000 & Rps4x \\
ENSMUST00000033741 & 198.11 & 785.72 & down & -1.99 & 0.0000 & Bgn \\
\hline
\end{tabular}

\subsection{QRT-PCR Validation}

Next, to validate our RNA-seq results, we selected three upregulated and three downregulated significant DE lncRNAs for qRT-PCR assay. Figure 4 shows that the qPCR data on the expression of selected lncRNAs coincided with our sequencing data, thereby indicating that our transcript identification and abundance estimation are highly reliable.

ENSMUST00000196219

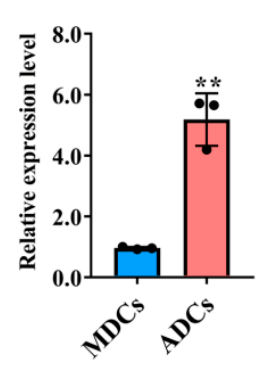

ENSMUST00000126640

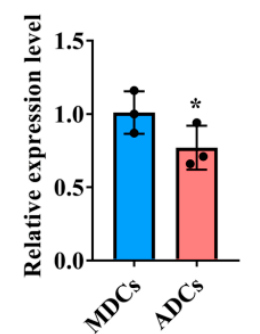

ENSMUST00000180396

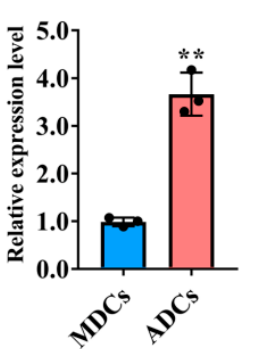

ENSMUST00000139288

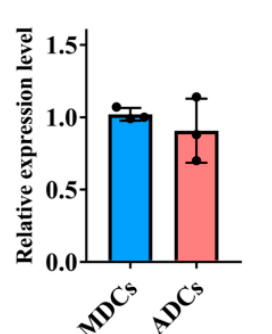

ENSMUST00000176549

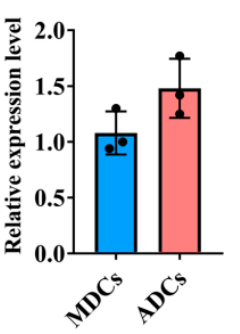

ENSMUST00000193796

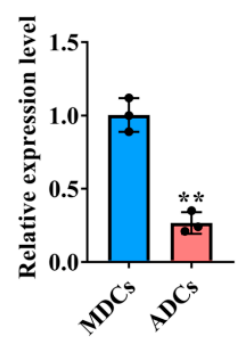

Figure 4. qPCR validation of the RNA-Sequencing (RNA-Seq) expression results of lncRNAs (six differentially expressed genes). The data are presented as the means \pm SEM. $n=3,{ }^{* *} P \leq 0.01$, * $P \leq 0.05$, Student's $t$-test.

\subsection{Functional Prediction of DE $\operatorname{lncRNAs}$}

We predicted the potential targets of these IncRNAs in cis- and trans- regulation to determine the functions of the 114 core DE lncRNAs. For the cis- regulation of lncRNAs, we searched for DE mRNAs that were situated $100 \mathrm{~kb}$ upstream and downstream of the lncRNAs. The RIsearch software was used to analyze the trans-regulated mRNAs, and the threshold of free energy was $<-100$. Finally, 728 trans target genes and 118 cis targets for 77 core lncRNAs were obtained.

The functions of these target genes were annotated by GO and KEGG pathway enrichment analyses (Figure 5). A total of $255 \mathrm{GO}$ entries with $P<0.05$ were obtained, and Figure 5A shows the top 30 GO entries with $P<0.01$. Moreover, several of the GO items with $P<0.05$ were related to muscle cell differentiation and muscle formation, including GO:0048747 (muscle fiber development), 
GO:0051145 (smooth muscle cell differentiation), GO:0016203 (muscle attachment), and GO:0042692 (muscle cell differentiation).

Figure 5B shows the results of enrichment analysis of KEGG signaling pathways. The target genes were involved in some of the signaling pathways responsible for lipid metabolism and energy homeostasis with $P<0.1$, such as the PPAR and mTOR pathways. In addition, some of the genes were involved in the MAPK $(P=0.3)$ and the JAK-STAT $(P=0.17)$ pathways, which are closely related to lipogenesis. These results suggest that DE lncRNAs and their target genes regulate the adipogenic transdifferentiation of muscle cells by regulating cell fate determination, intracellular gene reprogramming, cell differentiation, and substance metabolism.

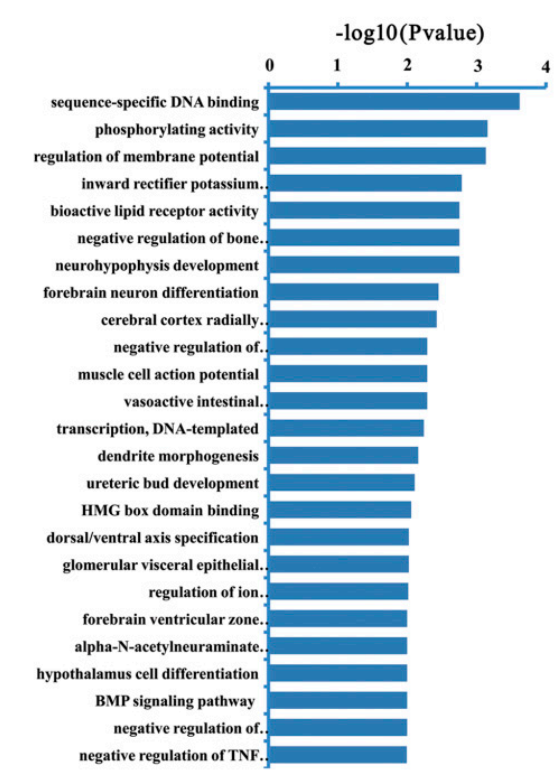

A

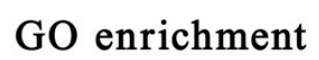

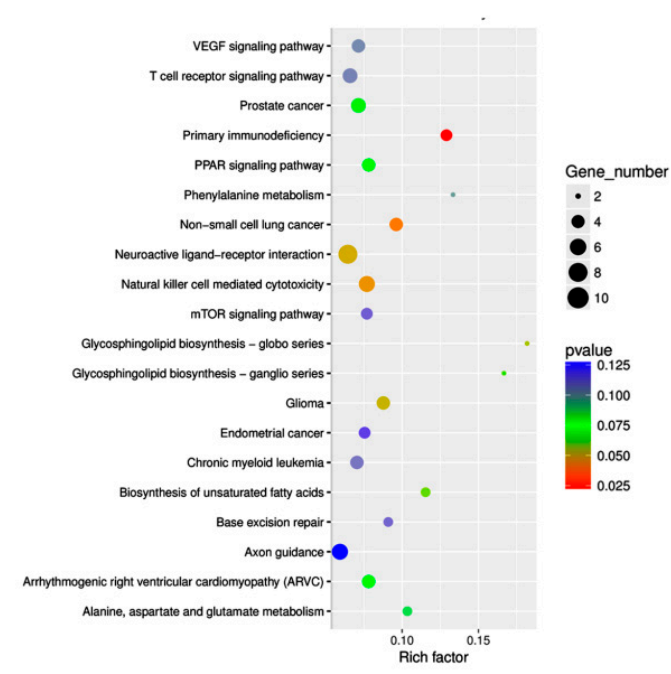

B KEEG enrichment

Figure 5. The top Gene Ontology (GO, (A)) and Kyoto Encyclopedia of Genes and Genomes (KEGG, (B)) enrichment analyses of the targets of differentially expressed lncRNAs.

In addition, several targets enriched the functional category of mesodermal cell fate determination (GO:0007500) (Figure 6). Both muscle and adipose cells originated from the common mesoderm cells in mammals, which thereby suggested that the corresponding lncRNAs could mediate the initial determination of cellular fate. Eight DE IncRNAs may thus possibly influence mesodermal cell fate by targeting BMP4, a member of the BMP family, which controls the differentiation of myoblasts as well as regulates adipogenesis in adipocytes $[10,29,30]$. The DGE-seq data also showed that BMP4 is upregulated in ADCs.

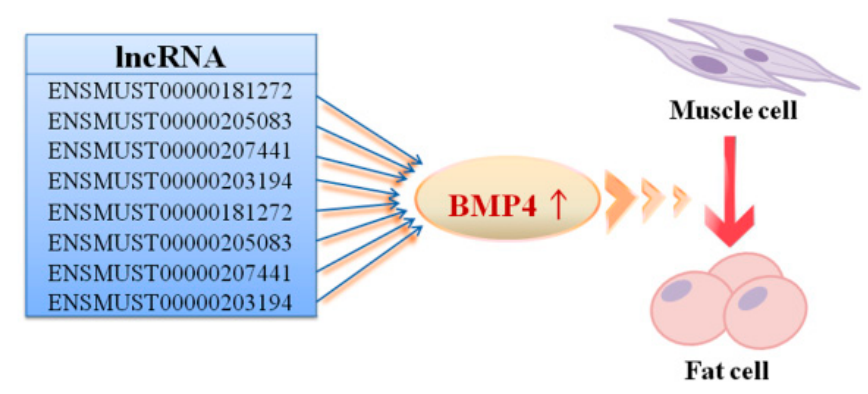

Figure 6. The target predication and GO analysis indicate that eight lncRNAs are related to cell fate decision in myoblasts based on the target gene, BMP4. 


\subsection{IncRNA-GM43652 Plays a Role in the Adipogenesis of C2C12 Cells}

In the present study, ENSMUST00000196219 (name: IncRNA-Gm43652) showed the highest expression level and the largest fold change in expression in ADCs as compared to MDCs. Therefore, we inferred that this gene might be directly related to cell adipogenesis. Additionally, the expression level of ENSMUST00000196219 at different time points during the adipogenesis of C2C12 cells was

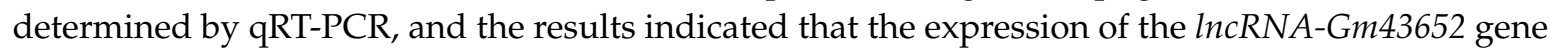
decreased during the early stages of transdifferentiation and was later consistently upregulated (Figure 7A). Furthermore, knocking down $\ln c R N A-G m 43652$ by siRNA transfection suppressed lipid

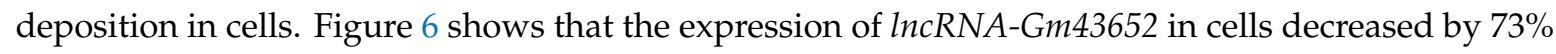
and $57 \% 24 \mathrm{~h}$ and $72 \mathrm{~h}$ after siRNA transfection as compared to the control cells (Figure 7B). Intracellular lipid deposition also decreased by approximately $30 \%$ (based on Oil Red O staining), and FABP4

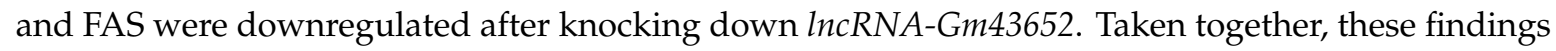
indicate that LncRNA-Gm43652 is a candidate regulator of adipogenic transdifferentiation of myoblasts.
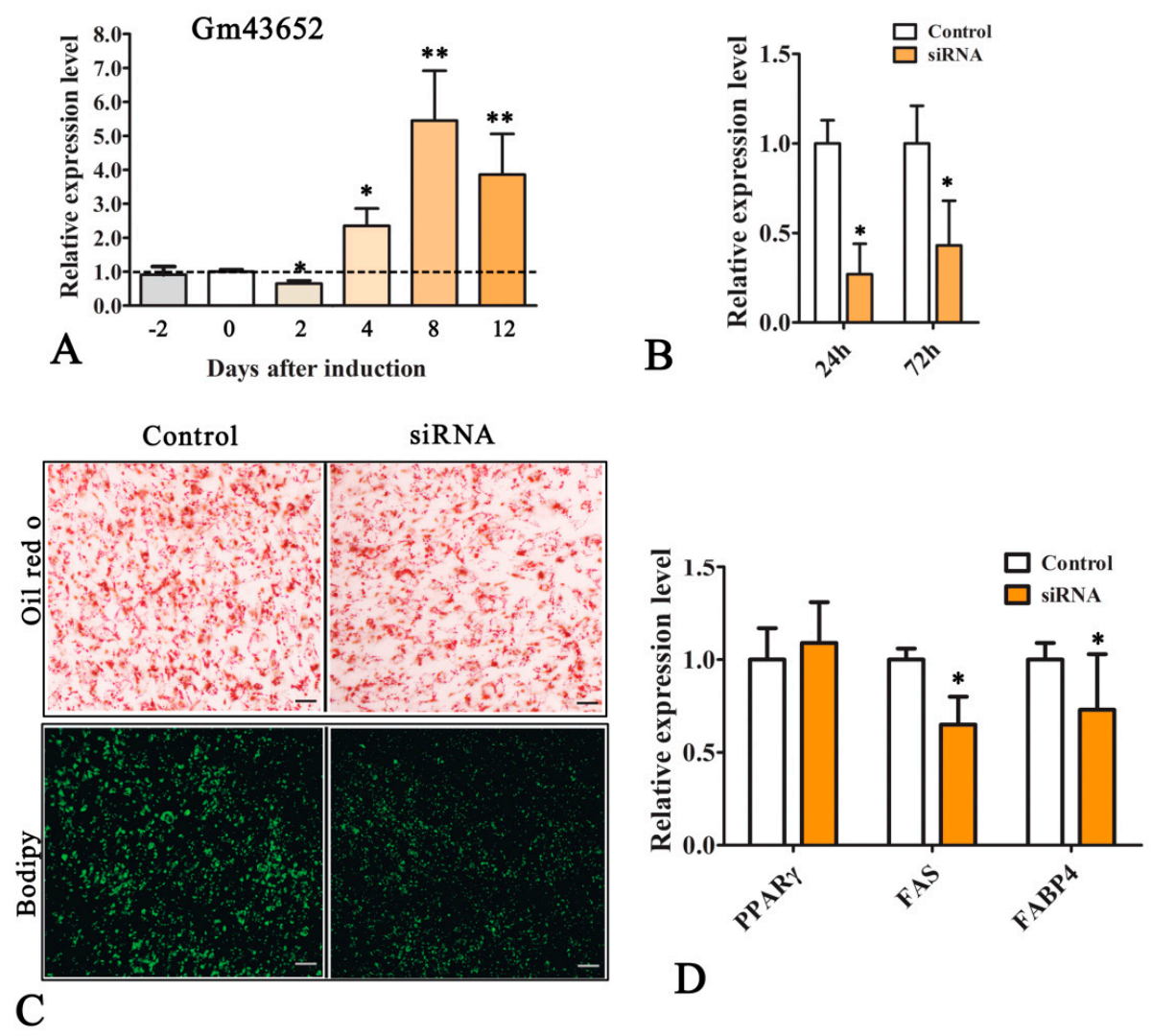

Figure 7. The $\ln R N A$-GM43652 gene is a potential regulator of the adipogenic conversion of $\mathrm{C} 2 \mathrm{C} 12$ myoblasts. (A) Changes in the expression of $I n c R N A-G M 43652$ during adipogenic transdifferentiation of myoblasts. (B) Knock down of lncRNA-GM43652 using small interfering RNAs (siRNAs). (C) Knocking

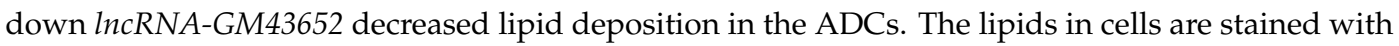
Oil Red O and BODIPY. (D) Knocking down lncRNA-GM43652 decreased the mRNA levels of the adipogenic genes. Expression levels were detected by qRT-PCR, $n=3$. The data are presented as the mean \pm SEM. ${ }^{*} P \leq 0.05$, student's $t$-test.

\section{Discussion}

The main function of skeletal muscle is to contract to facilitate movement of the body and to provide energy storage and expenditure. In addition, skeletal muscle is the largest endocrine organ in humans and animals that produces and releases many cytokines. These proteins that are released by skeletal muscle tissues could influence metabolism in other organs, such as adipose and liver [31]. 
Therefore, normal growth and the stability functions of skeletal muscle may influence the body's metabolic balance and energy homeostasis. Additionally, the growth and development of skeletal muscle also determines the meat production of livestock and the meat quality we can obtain [32].

IncRNAs have emerged as a class of important molecules that regulates gene expression at the epigenetic, transcriptional, and post-transcriptional levels through a wide array of mechanisms [33]. Numerous lncRNAs that are related to growth and development of muscle in humans and animals have been identified through sequencing and microarray projects. These lncRNAs participate in all of the processes of skeletal muscle development, including proliferation, differentiation, and fusion of myocytes, muscle hypertrophy, and conversion of muscle fiber types by targeting pro- or anti-myogenic genes. For example, lnc-MD1 could stimulate myogenesis and muscle cell differentiation by upregulating the expression of MEF2C and controlling transcriptional co-activator 1 by competitive binding with microRNAs, such as miR-135 and miR-133 [21].

The adipogenic conversion of muscle cells may change cellular fate when numerous genes are reprogrammed. Molecular regulation and metabolic physiology may change to address the requirements of adipogenesis during transdifferentiation. In recent years, we have gained new insights into the adipogenesis in muscle cells. To our knowledge, the formation of muscle, bone, and adipose tissues involves a multistep processes that includes the determination of a common progenitor mesodermal cell toward a specific differentiation pathway, followed by the expression of various terminal differentiation phenotypes [34]. In vitro studies have demonstrated the multi-directional differentiation potential of muscle-derived stem cells or precursor cells [1-6,35,36]. Our previous study has shown that some miRNAs participate in the control of adipogenesis in muscle cells, such as miR-199a, which negatively regulates the transdifferentiation of C2C12 myoblasts by targeting the FATP1 gene [15]. In this study, our analysis has also shown that several lncRNA genes are involved in the determination of cellular fate by affecting BMP4, which is a key molecular switch for myogenesis and adipogenesis. Additionally, KEGG pathway analysis of coding genes in the present study has revealed that the absorption and utilization of nutrients in the adipogenic and myogenic $\mathrm{C} 2 \mathrm{C} 12$ cells are different.

In addition, some reports have indicated that the fat cells transformed from muscle precursor cells exhibit the characteristics of brown adipocytes. Lineage-tracing experiments have shown that brown adipocytes, skeletal muscle cells, and dorsal dermal cells are all derived from the same multi-potential progenitor cells that originate from the central dermomyotome [37]. A study has revealed that Myf5-expressing progenitors can give rise to both skeletal muscle and brown fat cells [38]. The transcriptional regulator PRDM16 controls the switch of cell fate $[39,40]$. An et al. have also shown that the MyoD/Myf5-E2F4/p107/p130 axis functions as a molecular switch in postnatal myoblasts, which regulates the choice between differentiation into myoblasts and brown adipocytes [41]. We have also observed differences in lipid droplet morphology in adipogenic C2C12 cells compared to 3T3-L1 adipocytes using the similar induction conditions. UCP1 and PRDM16, two markers for brown adipocytes, have shown higher expression levels in adipogenic C2C12 cells than 3T3-L1 adipocytes, indicating that adipogenic myoblasts are more similar to brown adipocytes.

Although current knowledge on the mechanism of adipogenesis conversion of muscle cells is expanding, our understanding of the changes and function of lncRNAs in the transformation process remains limited. The present study has revealed huge differences in the gene expression profiles between normal myogenic differentiation and adipogenic transdifferentiation of myoblasts while using high-throughput RNA sequencing. A total of 1065 DE mRNA transcripts and 836 DE lncRNA transcripts between adipogenic cells and myogenic cells were identified in this study. Approximately 114 lncRNAs and 171 mRNAs were selected as core candidate regulators that are involved in the regulation of muscle cell transdifferentiation and subjected to further investigation.

We further predicted the target mRNAs for core lncRNAs while using cis and trans regulation analysis, and the results showed that there were 846 mRNAs for 77 lncRNAs that participate in the control of cell fate determination, intracellular gene reprogramming, cell differentiation, and substance 
metabolism. However, the biological functions of lncRNAs are highly diverse, and lncRNAs generally exert their effects on cells through different mechanisms. A number of studies have shown that lncRNAs can serve as molecular guides for chromatin-modifying complexes to their target genes, act as molecular signals in response to DNA damage, function as protein or miRNA decoys to regulate mRNA expression, and prevent binding to their targets or elements [42]. The sequencing results of the present study have revealed that several lncRNAs may be related to the adipogenic conversion of muscle cells. However, that is not sufficient for fully understanding the regulatory functions of lncRNAs in transformation.

The in-depth study and exploration on the molecular mechanism of adipogenesis in muscle cells will improve our understanding of the process of transformation between muscle and adipose, the metabolic functions of muscle tissues, and the pathogenesis of muscular diseases. Our present study utilized RNA sequencing to provide useful information regarding the changes in lncRNA expression during the adipogenesis of myoblasts. Furthermore, we have generated a list of candidate lncRNAs that are related to myoblast transdifferentiation that may be employed in future research studies.

\section{Materials and Methods}

\subsection{Cell Culture}

Mouse C2C12 myoblasts were resuscitated and cultured in Dulbecco's modified Eagle's medium (DMEM)-F12 medium (containing 10\% fetal calf serum and $100 \mathrm{U} / \mathrm{mL}$ penicillin/streptomycin; GIBCO, Grand Island, NY, USA) and then passaged when the confluency reached $90 \%$. The passaged cells were plated into six-well culture plates and then induced to undergo myogenic differentiation and adipogenic transdifferentiation. The myogenic differentiation was induced in subconfluent cells in differentiation medium (DMEM, $2 \%$ horse serum, $100 \mathrm{U} / \mathrm{mL}$ penicillin/streptomycin). The adipogenic trans-differentiation was inducted in adipogenesis medium (DMEM-F12, 20\% fetal calf serum, $10 \mu \mathrm{g} / \mathrm{mL}$ insulin, $1 \mu \mathrm{M}$ dexamethasone, $0.5 \mathrm{mM}$ 3-isobutyl-1-methylxanthine, and $10 \mu \mathrm{M}$ rosiglitazone, Sigma-Aldrich, St. Louis, MO, USA). The Giemsa staining and Oil red O staining (Solarbio, Shanghai, China) were used to determine the myogenesis and adipogenesis in different differentiated cells respectively as our previous describe [43]. All of the experiments were performed while using three separate cell clones.

\subsection{Oil Red $O$ and Giemsa Staining}

The status of adipogenic transdifferentiation and adipogenesis in $\mathrm{C} 2 \mathrm{C} 12$ cells were assessed by the Oil Red O method. Briefly, the cells were washed three times with phosphate buffered saline (PBS, $\mathrm{pH} 7.2$ ) and fixed in $4 \%$ paraformaldehyde for $30 \mathrm{~min}$ at ambient temperature. The fixed cells were washed three times in PBS again and then incubated in staining solution $(60 \%$ Oil Red O stock solution and $40 \% \mathrm{H}_{2} \mathrm{O}$ ) for $30 \mathrm{~min}$ at ambient temperature. The cells were washed twice with deionized water and observed under an inverted microscope.

During myogenic differentiation, the multinucleated myotubes were visualized in $\mathrm{C} 2 \mathrm{C} 12$ cell cultures with the Giemsa staining. Cells were washed twice with PBS, fixed in $4 \%$ paraformaldehyde for $30 \mathrm{~min}$, and then stained for $1 \mathrm{~min}$ with Giemsa stain solution (Solarbio, Beijing, China) at ambient temperature. Cells were washed twice with deionized water and observed under an inverted microscope.

\subsection{Sample Collection}

Undifferentiated cells (UDCs), cells that underwent myogenesis induction for five days (myogenic differentiated cells, MDCs), and cells subjected to adipogensis induction for eight days (adipogenic differentiated cells, ADCs) were separately collected for total RNA extraction. Three cell clones per treatment were pooled into one sequencing sample. 


\subsection{DGE-seq Sequencing and Data Processing}

Total RNA was extracted using TRIzol reagent (Invitrogen, CA, USA) while following the manufacturer's procedure. The total RNA quality and quantity were analysis of Bioanalyzer 2100 and RNA 6000 Nano Lab Chip Kit (Agilent, CA, USA) with RIN number $>7.0$. Approximately $10 \mu \mathrm{g}$ of total RNA was used to remove the ribosomal RNA according to the manuscript of the Epicentre Ribo-Zero Gold Kit (Illumina, San Diego, CA, USA). Following purification, the ribo-minus RNA fractions is fragmented into small pieces using divalent cations under elevated temperature. Subsequently, the cleaved RNA fragments were reverse-transcribed to create the final cDNA library in accordance with a strand-specific library preparation by dUTP method. Afterwards, we performed the single end sequencing on an Illumina HiSeq 2000/2500 at the LC Sciences (Hangzhou, China), following the vendor's recommended protocol.

The raw data containing adaptor sequences, tags with low quality sequences and unknown nucleotides $\mathrm{N}$ were filtered to obtain clean reads of $36 \mathrm{nt}$ length. The clean reads were then subjected to quality assessment. These included classification of total and distinct reads and assessment of their percentage in the library, analysis of saturation of the library, and correlation analysis of biological replicates. Bowtie mapped all clean tags to the transcripts sequence; only 1-bp mismatch is allowed. For monitoring the mapping events on both strands, both the sense and the complementary antisense sequences were included in the data collection. The number of perfect clean reads corresponding to each gene was calculated and normalized to the number of reads per kilobase of exon model per million mapped reads (RPKM).

\subsection{Analyses of Differential Expressed (DE) lncRNAs}

Based on the expression levels, significant DE lncRNAs among different cell samples were identified with $P$-value $\leq 0.05$ and $\log 2$ fold-change $(\log 2 \mathrm{FC}) \geq 1$. The cluster of the DEGs was performed while using common Perl and R scripts.

\subsection{Target Genes of DE lncRNAs and Enrichment Analysis}

The regulation of lncRNAs on target genes is mainly divided into two categories, namely cis and trans. The cis-regulatory targets of lncRNAs are predicted based on their positional relationships, defining DE lncRNAs and DE mRNAs within each 100-kb region of chromosomes. The target genes of lncRNAs exhibiting trans regulation are predicted mainly based on the free energy that is needed to form secondary structures between lncRNAs and mRNA sequences. In this study, the RNAplex (http://www.tbi.univie.ac.at/software/) algorithm was used to predict the trans targets of DE lncRNAs. Finally, all the cis- and trans-regulated genes were taken as the targets for the DE lncRNAs. Gene Ontology (GO) functional analysis was used to determine the main biological functions of the candidate target genes. KEGG metabolic pathway analysis identified the most important biochemical and signal transduction pathways that were involved in the candidate target genes.

\section{7. $q R T-P C R$}

Six DE lncRNAs were selected for qRT-PCR assay to verify the DGE-Seq results. In addition, the mRNA levels of several key adipogenic genes or myogenic genes were detected by qRT-PCR. The PCR detection was performed while using the Q6 qPCR system with SYBR Premix Ex Taq II (TaKaRa) and normalized using GAPDH as the endogenous control. All of the reactions were prepared while using three replicates and the expression levels of genes were expressed as fold change using the $2^{-\Delta \Delta \mathrm{CT}}$ method.

\subsection{SiRNA Transient Knockdown}

A siRNA of lncRNA-GM43652 and a nonspecific duplex (negative control) were custom synthesized by Biotech Co. (Nantong, China). The siRNA transfected into the adipogenic $\mathrm{C} 2 \mathrm{C} 12$ cells 
after four days of induction using Rfect siRNA Transfection Reagent (BIO-TRAN), according to the manufacturer's protocol. The cells were performed Oil red O staining, BODIPY staining, and qPCR analysis four days after transfection.

\subsection{Statistical Analysis}

The data were processed while using R, Microsoft Excel, and GraphPad Prism 5.0. Differences among groups were analyzed using one-way analyses of variance (ANOVAs), followed by Dunnett's test. Two-tailed student's $t$-test was used for comparison between two groups, and $P<0.05$ was considered to be statistically significant.

Supplementary Materials: The following are available online at http://www.mdpi.com/1422-0067/20/15/3725/s1.

Author Contributions: R.Q. and F.Y. designed the experiments; X.Q., Q.W., and J.W. performed the experiments; F.Y. and J.H. administrated project; Y.Z. analyzed the data; R.Q., X.Q., and M.W. wrote the paper. All authors read and approved the final manuscript.

Funding: This research was funded by the Fundamental and Frontier Research Project of Chongqing (Project Number: cstc2017jcyjBX0023 and cstc2018jxj10017) and the National Key R\&D Program of China (2018YFD0500404).

Acknowledgments: We would like to thank LetPub (www.letpub.com) for providing linguistic assistance during the preparation of this manuscript.

Conflicts of Interest: The authors declare that they have no competing interests.

\section{Abbreviations}

$\begin{array}{ll}\text { ADCs } & \text { Adipogenic differentiated cells } \\ \text { BMP4 } & \text { The growth factors bone morphogenetic protein-4 } \\ \text { FAS } & \text { Fatty acid synthase } \\ \text { GO } & \text { Gene ontology } \\ \text { KEEG } & \text { Kyoto encyclopedia of genes and genomes } \\ \text { LncRNA } & \text { Long chain non-coding RNA } \\ \text { MYF5 } & \text { Myogenic factor } 5 \\ \text { MDCs } & \text { Myogenic differentiated cells } \\ \text { PPAR } \gamma & \text { Peroxisome proliferator-activated receptor gama } \\ \text { PRDM16 } & \text { PRD1-BF1-RIZ1 homologous domain containing 16 } \\ \text { RPKM } & \text { Reads Per Kilobase of exon modelper Million mapped reads } \\ \text { siRNA } & \text { Small interfering RNA } \\ \text { UCP1 } & \text { Uncoupling protein 1 } \\ \text { UDCs } & \text { Undifferentiated cells }\end{array}$

\section{References}

1. Hu, E.; Tontonoz, P.; Spiegelman, B.M. Transdifferentiation of myoblasts by the adipogenic transcription factors PPAR $\gamma$ and C/EBP $\alpha$. Proc. Natl. Acad. Sci. USA 1995, 92, 9856-9860. [CrossRef] [PubMed]

2. Teboul, L.; Gaillard, D.; Staccini, L.; Inadera, H.; Amri, E.Z.; Grimaldi, P.A. Thiazolidinediones and fatty acids convert myogenic cells into adipose-like cells. J. Biol. Chem. 1995, 270, 28183-28187. [PubMed]

3. Asakura, A.; Komaki, M.; Rudnicki, M.A. Muscle satellite cells are multipotential stem cells that exhibit myogenic, osteogenic, and adipogenic differentiation. Differentiation 2001, 68, 245-253. [CrossRef] [PubMed]

4. Yeow, K.; Phillips, B.; Dani, C.; Cabane, C.; Amri, E.Z.; Derijard, B. Inhibition of myogenesis enables adipogenic trans-differentiation in the $\mathrm{C} 2 \mathrm{C} 12$ myogenic cell line. FEBS Lett. 2001, 506, 157-162. [CrossRef]

5. Jiang, J.; Zhou, P.; Ling, H.; Xu, Z.Z.; Yi, B.; Zhu, S.H. MiR-499/PRDM16 axis modulates the adipogenic differentiation of mouse skeletal muscle satellite cells. Hum. Cell 2018, 31, 282-291. [CrossRef] [PubMed]

6. Kook, S.H.; Choi, K.C.; Son, Y.O.; Lee, K.Y.; Hwang, I.H.; Lee, H.J.; Chang, J.S.; Choi, I.H.; Lee, J.C. Satellite cells isolated from adult hanwoo muscle can proliferate and differentiate into myoblasts and adipose-like cells. Mol. Cells 2006, 22, 239-245. [PubMed] 
7. Coppi, D.P.; Milan, G.; Scarda, A.; Boldrin, L.; Centobene, C.; Piccoli, M.; Pozzobon, M.; Pilon, C.; Pagano, C.; Gamba, P.; et al. Rosiglitazone modifies the adipogenic potential of human muscle satellite cells. Diabetologia 2006, 49, 1962-1973. [CrossRef] [PubMed]

8. Rosen, E.D.; Spiegelman, B.M. Molecular regulation of adipogenesis. Annu. Rev. Cell Dev. Biol. 2000, 16, 145-171. [CrossRef]

9. Lexell, J. Human aging, muscle mass, and fiber type composition. J. Gerontol. A Biol. Sci. Med. Sci. 1995, 50, 11-16.

10. Plikus, M.V.; Guerrero-Juarez, C.F.; Ito, M.; Li, Y.R.; Dedhia, P.H.; Zheng, Y.; Shao, M.; Gay, D.L.; Ramos, R.; Hsi, T.C.; et al. Regeneration of fat cells from myofibroblasts during wound healing. Science 2017, 355, 748-752. [CrossRef]

11. Ma, J.; Wang, H.; Liu, R.; Jin, L.; Tang, Q.; Wang, X.; Jiang, A.; Hu, Y.; Li, Z.; Zhu, L.; et al. The miRNA transcriptome directly reflects the physiological and biochemical differences between red, white, and intermediate muscle fiber types. Int. J. Mol. Sci. 2015, 16, 9635-9653. [CrossRef] [PubMed]

12. Memczak, S.; Jens, M.; Elefsinioti, A.; Torti, F.; Krueger, J.; Rybak, A.; Maier, L.; Mackowiak, S.D.; Gregersen, L.H.; Munschauer, M.; et al. Circular RNAs are a large class of animal RNAs with regulatory potency. Nature 2013, 495, 333-338. [CrossRef] [PubMed]

13. Li, J.; Tian, H.; Yang, J.; Gong, Z. Long noncoding RNAs regulate cell growth, proliferation, and apoptosis. DNA Cell Biol. 2016, 35, 459-470. [CrossRef] [PubMed]

14. Wang, H.; Li, X.; Gao, S.; Sun, X.; Fang, H. Transdifferentiation via transcription factors or microRNAs: Current status and perspective. Differentiation 2015, 90, 69-76. [CrossRef] [PubMed]

15. Qi, R.; Long, D.; Wang, J.; Wang, Q.; Huang, X.; Cao, C.; Gao, G.; Huang, J. MicroRNA-199a targets the fatty acid transport protein 1 gene and inhibits the adipogenic trans-differentiation of $\mathrm{C} 2 \mathrm{C} 12$ myoblasts. Cell. Physiol. Biochem. 2016, 39, 1087-1097. [CrossRef] [PubMed]

16. Lee, E.J.; Lee, H.J.; Kamli, M.R.; Pokharel, S.; Bhat, A.R.; Lee, Y.H.; Choi, B.H.; Chun, T.; Kang, S.W.; Lee, Y.S.; et al. Depot-specific gene expression profiles during differentiation and transdifferentiation of bovine muscle satellite cells, and differentiation of preadipocyte. Genomics 2012, 100, 195-202. [CrossRef] [PubMed]

17. Tsai, M.C.; Manor, O.; Wan, Y.; Mosammaparast, N.; Wang, J.K.; Lan, F.; Shi, Y.; Segal, E.; Chang, H.Y. Long noncoding RNA as modular scaffold of histone modification complexes. Science 2010, 329, 689-693. [CrossRef]

18. Huarte, M.; Rinn, J.L. Large non-coding RNAs: Missing links in cancer? Hum. Mol. Genet. 2010, 19, R152-R161. [CrossRef]

19. Guttman, M.; Rinn, J.L. Modular regulatory principles of large non-coding RNAs. Nature 2012, 482, 339-346. [CrossRef]

20. Gong, C.; Li, Z.; Ramanujan, K.; Clay, I.; Zhang, Y.; Lemire-Brachat, S.; Glass, D.J. A Long non-coding RNA, LncMyoD, regulates skeletal muscle differentiation by blocking IMP2-mediated mRNA translation. Dev. Cell 2015, 34, 181-191. [CrossRef]

21. Cesana, M.; Cacchiarelli, D.; Legnini, I.; Santini, T.; Sthandier, O.; Chinappi, M.; Tramontano, A.; Bozzoni, I. A long noncoding RNA controls muscle differentiation by functioning as a competing endogenous RNA. Cell 2011, 147, 358-369. [CrossRef] [PubMed]

22. Zhou, L.; Sun, K.; Zhao, Y.; Zhang, S.; Wang, X.; Li, Y.; Lu, L.; Chen, X.; Chen, F.; Bao, X.; et al. Linc-YY1 promotes myogenic differentiation and muscle regeneration through an interaction with the transcription factor YY1. Nat. Commun. 2015, 6, 10026. [CrossRef] [PubMed]

23. Sun, L.; Goff, L.A.; Trapnell, C.; Alexander, R.; Lo, K.A.; Hacisuleyman, E.; Sauvageau, M.; Tazon-Vega, B.; Kelley, D.R.; Hendrickson, D.G.; et al. Long noncoding RNAs regulate adipogenesis. Proc. Natl. Acad. Sci. USA 2013, 110, 3387-3392. [CrossRef] [PubMed]

24. Cai, R.; Tang, G.; Zhang, Q.; Yong, W.; Zhang, W.; Xiao, J.; Wei, C.; He, C.; Yang, G.; Pang, W. A novel lnc-RNA, named lnc-ora, is identified by RNA-seq analysis, and its knockdown inhibits adipogenesis by regulating the PI3K/AKT/mTOR signaling pathway. Cells 2019, 8, 477. [CrossRef] [PubMed]

25. Yi, F.; Zhang, P.; Wang, Y.; Xu, Y.; Zhang, Z.X.; Ma, W.Z.; Xu, B.; Xia, Q.; Du, Q. Long non-coding RNA slincRAD functions in methylation regulation during the early stage of mouse adipogenesis. RNA Biol. 2019, 19, 1-13. [CrossRef] [PubMed] 
26. Litwiniuk, A.; Pijet, B.; Pijet-Kucicka, M.; Gajewska, M.; Pająk, B.; Orzechowski, A. FOXO1 and GSK-3 $\beta$ are main targets of insulin-mediated myogenesis in C2C12 muscle cells. PLoS ONE 2016, 11, e0146726. [CrossRef] [PubMed]

27. Sun, F.; Fang, P.; Li, J.; Du, L.; Lan, Y.; Zhou, T.; Fan, Y.; Shen, W.; Zhou, Y. RNA-seq-based digital gene expression analysis reveals modification of host defense responses by rice stripe virus during disease symptom development in Arabidopsis. Virol. J. 2016, 13, 202. [CrossRef] [PubMed]

28. Niu, Y.Q.; Hu, B.; Li, Q.; Chen, H.B.; Takáč, T.; Šamaj, J.; Xu, C.X. Comparative digital gene expression analysis of tissue-cultured plantlets of highly resistant and susceptible banana cultivars in response to Fusarium oxysporum. Int. J. Mol. Sci. 2018, 19, 350. [CrossRef] [PubMed]

29. Sartori, R.; Schirwis, E.; Blaauw, B.; Bortolanza, S.; Zhao, J.; Enzo, E.; Stantzou, A.; Mouisel, E.; Toniolo, L.; Ferry, A.; et al. BMP signaling controls muscle mass. Nat. Genet. 2013, 45, 1309-1318. [CrossRef]

30. Gustafson, B.; Hammarstedt, A.; Hedjazifar, S.; Hoffmann, J.M.; Svensson, P.A.; Grimsby, J.; Rondinone, C.; Smith, U. BMP4 and BMP antagonists regulate human white and beige adipogenesis. Diabetes 2015, 64, 1670-1681. [CrossRef]

31. Finelli, C.; Tarantino, G. Have guidelines addressing physical activity been established in nonalcoholic fatty liver disease? World. J. Gastroenterol. 2012, 18, 6790-6800. [CrossRef] [PubMed]

32. Zhang, Y.; Yu, B.; Yu, J.; Zheng, P.; Huang, Z.; Luo, Y.; Luo, J.; Mao, X.; Yan, H.; He, J.; et al. Butyrate promotes slow-twitch myofiber formation and mitochondrial biogenesis in finishing pigs via inducing specific microRNAs and PGC-1 $\alpha$ expression. J. Anim. Sci. 2019. [CrossRef]

33. Ren, C.F.; Deng, M.T.; Fan, Y.X.; Yang, H.; Zhang, G.M.; Feng, X.; Li, F.; Wang, D.; Wang, F.; Zhang, Y. Genome-Wide analysis reveals extensive changes in lncrnas during skeletal muscle development in hu sheep. Genes 2017, 8, 191. [CrossRef] [PubMed]

34. Frasch, M. Dedifferentiation, redifferentiation, and transdifferentiation of striated muscles during regeneration and development. Curr. Top. Dev. Biol. 2016, 106, 331-355.

35. Buckingham, M. Gene regulatory networks and cell lineages that underlie the formation of skeletal muscle. Proc. Natl. Acad. Sci. USA 2017, 114, 5831-5837. [CrossRef] [PubMed]

36. Aguiari, P.; Leo, S.; Zavan, B.; Vindigni, V.; Rimessi, A.; Bianchi, K.; Franzin, C.; Cortivo, R.; Rossato, M.; Vettor, R.; et al. High glucose induces adipogenic differentiation of muscle-derived stem cells. Proc. Natl. Acad. Sci. USA 2008, 105, 1226-1231. [CrossRef]

37. Atit, R.; Sgaier, S.K.; Mohamed, O.A.; Taketo, M.M.; Dufort, D.; Joyner, A.L.; Niswander, L.; Conlon, R.A. Beta-catenin activation is necessary and sufficient to specify the dorsal dermal fate in the mouse. Dev. Biol. 2006, 296, 164-176. [CrossRef]

38. Lepper, C.; Fan, C.M. Inducible lineage tracing of Pax7-descendant cells reveals embryonic origin of adult satellite cells. Genesis 2010, 48, 424-436. [CrossRef]

39. Seale, P.; Bjork, B.; Yang, W.; Kajimura, S.; Chin, S.; Kuang, S.; Scimè, A.; Devarakonda, S.; Conroe, H.M.; Erdjument-Bromage, H.; et al. PRDM16 controls a brown fat/skeletal muscle switch. Nature 2008, 454, 961-967. [CrossRef]

40. Yin, H.; Pasut, A.; Soleimani, V.D.; Bentzinger, C.F.; Antoun, G.; Thorn, S.; Seale, P.; Fernando, P.; van Ijcken, W.; Grosveld, F.; et al. MicroRNA-133 controls brown adipose determination in skeletal muscle satellite cells by targeting prdm16. Cell Metab. 2013, 17, 210-224. [CrossRef]

41. An, Y.; Wang, G.; Diao, Y.; Long, Y.; Fu, F.; Weng, M.; Zhou, L.; Sun, K.; Cheung, T.H.; Ip, N.Y.; et al. A molecular switch regulating cell fate choice between muscle progenitor cells and brown adipocytes. Dev. Cell 2017, 41, 382-391. [CrossRef] [PubMed]

42. Klinge, C.M. Non-coding RNAs: Long non-coding RNAs and microRNAs in endocrine-related cancers. Endocr. Relat. Cancer 2018, 25, 259-282. [CrossRef] [PubMed]

43. Qi, R.; Liu, H.; Wang, Q.; Wang, J.; Yang, F.; Long, D.; Huang, J. Expressions and regulatory Effects of P38/ERK/JNK mapks in the adipogenic trans-differentiation of C2C12 myoblasts. Cell. Physiol. Biochem. 2017, 44, 2467-2475. [CrossRef] [PubMed]

(C) 2019 by the authors. Licensee MDPI, Basel, Switzerland. This article is an open access article distributed under the terms and conditions of the Creative Commons Attribution (CC BY) license (http://creativecommons.org/licenses/by/4.0/). 